\title{
Mental turmoil, suicide risk, illness perception, and temperament, and their impact on quality of life in chronic daily headache
}

\author{
Sergio De Filippis · Denise Erbuto · Federica Gentili • \\ Marco Innamorati · David Lester · Roberto Tatarelli · \\ Paolo Martelletti · Maurizio Pompili
}

Received: 17 July 2008/ Accepted: 2 September 2008/Published online: 25 October 2008

(C) Springer-Verlag 2008

\begin{abstract}
To evaluate the relationship among quality of life, temperament, illness perception, and mental turmoil in patients affected by chronic daily headache with concomitant medication overuse headache. Participants were 116 consecutive adult outpatients admitted to the Department of General Medicine of the Sant'Andrea Hospital in Rome, between January 2007 and December 2007 with a diagnosis of chronic daily headache (illness duration $>5$ years). Patients were administered the Temperament Evaluation of Memphis, Pisa, Paris and San Diego-autoquestionnaire version (TEMPS-A), the Beck Hopelessness Scale (BHS), the Hamilton Rating Scale for Depression (HAM-D), the Mini-International Neuropsychiatric Interview (MINI), the Revised Illness Perception Questionnaire (IPQ), the Suicide Score Scale (SSS), and the Quality of Life Index (QL-Index). Twenty-eight percent of the patients evidenced moderate to severe depression, and 35\% evidenced severe hopelessness. Analyses also indicated that quality of
\end{abstract}

S. De Filippis $(\varangle) \cdot$ P. Martelletti

Department of Medical Sciences, 2nd School of Medicine,

Sant'Andrea Hospital, Sapienza University of Rome,

Via di Grottarossa 1035, 00189 Rome, Italy

e-mail: sergio.defilippis@uniroma1.it

D. Erbuto - F. Gentili · R. Tatarelli - M. Pompili

Department of Psychiatry, 2nd School of Medicine,

Sant'Andrea Hospital, Sapienza University of Rome,

Rome, Italy

M. Innamorati

European University of Rome, Rome, Italy

D. Lester

The Richard Stockton College of New Jersey, Pomona, NJ, USA

M. Pompili

McLean Hospital, Harvard Medical School, Belmont, MA, USA life, temperament, illness perception, and psychological turmoil are associated. However, a hierarchical multivariate regression analysis with quality of life as dependent variable indicated that only a model with mental turmoil variables may fit data; further, only the MINI suicidal intent resulted associated with quality of life (standardized regression coefficient $=-0.55 ; \quad t=-3.06 ; \quad P<0.01$ ). Suicide risk may play a central role in affecting the quality of life of patients with chronic headache. The investigation of the interplay of factors that precipitate suicide risk should include assessment of chronic headache and its effects on wellbeing.

Keywords Chronic daily headache .

Medication overuse headache - Self-injurious behaviour . Suicide · Depression · Hopelessness - Quality of life

\section{Introduction}

The International Classification of Headache Disorders, II version (ICHD-II) recognises 24 types of chronic headache and defines primary episodic headaches as chronic when the attacks appear for more than 15 days/month and for at least 3 months [1]. The influence of psychological factors in the pathogenesis, clinical course and treatment of primary headache has always been of interest and is highly relevant for both research and clinical practice [2], especially since psychological and behavioural states seem particularly important in promoting its transformation into chronic daily headache $(\mathrm{CDH})$, which often sustains drug abuse [3].

Migraine may be combined with drug overuse, first labelled by ICDH-II in 2004 (and revised in 2005) as medication overuse headache $(\mathrm{MOH})$, when it is associated with overuse of a combination of analgesics, barbiturates, 
opioids, Ergot alkaloids, aspirin, FANS, caffeine and triptans. $\mathrm{MOH}$ has been the subject of increasing research attention in recent years. A negative impact of this type of headache has been detected on the quality of life (including professional, social and emotional aspects) and on psychiatric comorbidity [4]. Boes and Capobianco [5] documented the crucial role of depression in the process of transformation of migraine into MOH. Similar results were obtained by Radat et al. [6] in a retrospective comparative study carried out on 41 patients with migraine and 41 patients with $\mathrm{MOH}$ (treated with one or more of the following drugs, alone or in combination: paracetamol, codeine, tryptans, non-steroidal anti-inflammatory drugs [NSAID], salicylates, and ergot derivatives). They found a higher prevalence of anxiety and mood disorders in patients with $\mathrm{MOH}$ (as assessed through the DSM-IV MINI interview) during the phase of transformation of migraine into $\mathrm{MOH}$. A study by De Filippis et al. [7] showed that patients with chronic daily headache and those with $\mathrm{MOH}$ both show high levels of anxiety and depressive symptoms associated with alexithymia. Furthermore, the same study showed that anxiety and depression facilitated the onset of headache whereas, in some patients, the persistence of the pain rendered the patient more vulnerable to the onset of psychiatric symptoms.

Patients with headache who abuse analgesic drugs have an ambivalent attitude towards the drugs they take, similar to what occurs in drug addiction. In fact, it was recently proposed to frame $\mathrm{MOH}$ within the spectrum of addictive disorders [8].

The hypothesis of a greater psychological vulnerability of the patient with headache to daily hassles has been consistently confirmed $[9,10]$. This vulnerability is characterized by

(a) ineffective coping styles (internal coping, social avoidance and isolation, pessimism, poor perception of event predictability or control), and

(b) reduced affective regulation ability, in particular anger, towards which the patient with headache shows excessive inhibition-repression and/or inward directedness $[9,10]$.

In patients with chronic tension headache and facial pain, Jacobson and Folstein [11] observed inadequate stress coping mechanisms, along with major depression, panic attacks and psychoactive substance abuse.

Wacogne et al. [12] found clinically significant anxiety, as assessed through the Hospital Anxiety and Depression Scale, and emotional distress, as assessed through the Perceived Stress Questionnaire, in 141 patients with migraine compared to 109 controls, thereby confirming the role of stress as a trigger.

However, the higher stress vulnerability hypothesis of headache is not always confirmed. Peripheral stress markers may not correlate with stress in patients with headache and, despite the fact that many mechanisms may be shared among different headache sufferers, different headache subtypes may differ as to their stress vulnerability [13-15].

In Greco-Roman medicine, temperaments were proposed as constitutional emotional reactivity patterns fashioned by the humoral theory [16, 17]. Kraepelin [18] proposed four basic affective dispositions-depressive, manic, irritable and cyclothymic — which he believed to be subclinical forms of affective psychoses which had their roots in adolescence. In line with these classic formulations, and concordant with Kretschmer's [19] views, research by Akiskal and his colleagues [20,21] formulated criteria for temperaments which are relevant for mood disorders conceptualized in a continuum, ranging from subthreshold affective traits at one end to severe affective psychosis at the other end [17, 22, 23]. In this framework, five temperaments are proposed [24]: Depressive, Hyperthymic (borrowing this term from Schneider [25] to replace the 'manic' type of Kraepelin), Cyclothymic, Irritable and Generalized Anxious; the latter represents an addition beyond the classic German concepts [26]. However, it remains unresolved whether the Irritable temperament is merely a subtype of the Cyclothymic temperament or whether it stands on its own $[26,27]$. In addition, the placement of the anxious subtype within the classical temperaments remains to be confirmed by empirical data.

Investigating the affective temperament profiles of 150 nonviolent suicide attempters (121 of whom had a current major depressive episode) and 717 healthy controls, recent findings showed that the suicide attempters scored significantly higher on the four of the five affective temperaments (depressive, cyclothymic, irritable and anxious) [28].

The aim of the present study was to evaluate the relationship between the quality of life, temperament, illness perception, and mental turmoil in patients affected by chronic headache. Mental turmoil is inversely related to emotional well-being, a measure of "good life" as the individual views it. In the present study, mental turmoil was assessed using measures of depression and hopelessness. Suicidal intent was measured both with the specific section of the Mini-International Neuropsychiatric Interview (MINI), a DSM-IV-TR based, short, structured interview [29], and with the suicide score scale (SSS).

\section{Methods}

Study site and participants

Participants were 116 consecutive adult outpatients (102 women and $14 \mathrm{men}$ ) interviewed for clinical variables, 
admitted to the Regional Referral Headache Centre, Department of Medical Sciences, Sant'Andrea Hospital in Rome between January 2007-December 2007. Inclusion criteria were a diagnosis of $\mathrm{CDH}$ (illness duration $>5$ years) with concomitant $\mathrm{MOH}$, and an age $\geq 18$ years. Exclusion criteria were comorbidity with major disorders of the central nervous system (e.g., Parkinson's disease, dementia, epilepsy), delirium and any condition affecting the patient's ability to complete the assessment, including refusal of informed consent. The average age of the participants was 48.11 years $(\mathrm{SD}=12.03)$.

Participants participated voluntarily in the study, and each subject provided written informed consent. The study protocol received ethics approval from the local research ethics review board.

\section{Data collection and measurements}

Patients were administered the Temperament Evaluation of Memphis, Pisa, Paris and San Diego-autoquestionnaire version (TEMPS-A), the Beck Hopelessness Scale (BHS), the Hamilton Rating Scale for Depression (HAM-D) [30, 31], the MINI, the Revised Illness Perception Questionnaire (IPQ) [32], the SSS, and the Quality of Life Index (QL-Index) [33].

The TEMPS-A is a self-rating questionnaire composed of 109 items for men and 110 for women. The scoring of the items produces five scores for affective temperaments: Dysthymic, Cyclothymic, Anxious, Irritable and Hyperthymic. The Italian validation study of the TEMPS-A [34] indicated a 3-factor solution: a large factor representing the Dysthymic, Cyclothymic, and Anxious traits (Dys-CycAnx); the second and third factor replicated the original Irritable and the Hyperthymic temperaments. Cronbach Alpha coefficients for the three subscales were, respectively, 0.89, 0.77 and 0.74 .

The BHS is a 20 -item scale for measuring the cognitive component of the syndrome of depression. Hopelessness is a cognitive trait considered to be associated with suicide, leading suicidal patients to believe that suicide is the only feasible strategy for dealing with their seemingly insoluble problems. Empirical research has indicated a relationship between hopelessness and suicidal intent [35-37]. Beck et al. $[38,39]$ carried out two prospective studies of inpatient and outpatient samples. The authors found that hopelessness scores were related significantly to eventual completed suicide. A cut-off score of 9 or above identified most of the patients who eventually committed suicide. The high-risk group identified in the latter study was 11 times more likely to commit suicide than the rest of the outpatients. Thus, hopelessness may be used as an indicator of suicide potential. Italian validation of the Beck Hopelessness Scale was provided by Pompili et al. [40] which confirmed the existence of a strong association between hopelessness and suicide risk.

The HAM-D is a 21 -item scale rating the severity of depressive symptoms during the previous 7 days. The scale assesses autonomic, vegetative and psychological symptoms of depression. Studies have reported adequate convergent validity and internal reliability [41]. A cut-off of 15 or above is associated with moderate to severe depression.

The MINI was used to evaluate suicide intent. The section assessing the suicide intent evaluates the past and current suicide behaviour and ideation and classifies subjects into four groups: no suicidal risk, low suicidal risk, moderate suicidal risk, and high suicidal risk. For the present study, we grouped subjects into two groups: lower suicidal intent (no suicidal intent and low suicidal intent) and higher suicidal intent (moderate suicidal intent and high suicidal intent).

The SSS consists of two sections, assessing suicide ideation and behaviour in the last 12 months and lifetime excluding the last year. Both sections consisted of six truefalse items: "Feel tired of living" (item 1), "Think that for you, for your family and friends, it would better to be dead" (item 2), "Think about physically hurting yourself" (item 3), "Think about taking your own life" (item 4), "Have planned a way of taking your own life" (item 5), and "Have tried to take your own life" (item 6). Although, the Suicide Score Scale has not yet been fully evaluated, it has been previously administered to 851 Italian undergraduate students with good internal consistency (Cronbach alpha 0.87 and an inter-item mean correlation of 0.35 ) and moderate convergent validity with measures assessing depression, reasons for living and aggression (Reasons for Living total score: $r=-0.32 ; P<0.001$; Zung Depression Scale: $r=-0.41 ; P<0.001$; Aggression Questionnaire: $r=-0.53 ; P<0.001)$.

The IPQ measures patients' illness perceptions. It has demonstrated good reliability and validity across several illness groups. The IPQ includes eight dimensions. Illness identity measures the number of commonly experienced symptoms such as pain, nausea, and upset stomach that the patient associates with his/her particular illness. Consequences measures patients' beliefs about the seriousness of their condition. Timeline is divided into an acute/chronic subscale and a cyclical subscale that assesses whether patients see their illness as cyclical in nature. The control dimension is divided into personal control, which refers to beliefs about one's own ability to control symptoms, and treatment control, which refers to a belief that treatment is an effective way of controlling the illness. The illness coherence subscale measures the degree to which patients feel they have a coherent understanding or model of their condition. Finally, the items of the causal dimension can be 
divided into four main subscales: psychological attributions that include items such as stress and overwork, risk attributions that include factors such as diet and heredity, immune attributions that include factors such as a virus and the immune system, and chance attributions such as an accident or bad luck.

The QL-index is a short self-administered scale evaluating five aspects of quality of life: activity, daily living, health, support and outlook, with a choice of three possible answers and with higher scores reflecting a better quality of life.

Data analysis

Pearson's correlations were calculated to test the relationship among quality of life, illness perception, temperament, and mental turmoil scores. Hierarchical multivariate regression analysis was performed to test multivariate associations among the variables.

All statistical analyses were performed using the SPSS 13.0 statistical software package.

\section{Results}

Summary statistics are listed in Table 1 . Twenty-eight percent of the $\mathrm{CDH}$ plus $\mathrm{MOH}$ patients had a score on the HAM-D of 15 or above indicating a moderate to severe depression; 35\% reported a BHS score of 9 or above indicating severe hopelessness, and around $8 \%$ were evaluated with moderate to severe suicide intent on the MINI. Thus, one out of three patients reported clinically relevant symptoms of depression and hopelessness, although the incidence of patients considered being at risk of suicide was low.

Pearson's correlations between the variables are shown in Table 2. The results indicate that quality of life, temperament, illness perception, and psychological turmoil are associated. Quality of life is significantly associated with TEMPS Dys-Cyc-Anx $(r=-0.27 ; P<0.05)$, depression $(r=-0.61 ; \quad P<0.01), \quad$ hopelessness $\quad(r=-0.34$; $P<0.01$ ), SSS scores (Part 1: $r=-0.47 ; P<0.01$. Part 2: $r=-0.32 ; P<0.01$. Total score: $r=-0.41$; $P<0.01)$, and the symptoms $(r=-0.29 ; P<0.05)$ and emotional representation $(r=-0.34 ; P<0.01)$ dimensions of the IPQ. Temperament was associated with several illness perception dimensions: hyperthymic temperament was inversely related to consequence $(r=-0.28$; $P<0.01)$ and directly related to personal control $(r=0.27 ; \quad P<0.01)$; irritability was associated with timeline acute/chronic $(r=0.21 ; P<0.05)$ and with emotional representation $(r=0.28 ; P<0.01)$; Dys-CycAnx was related to symptoms $(r=0.40 ; P<0.01)$,
Table 1 Descriptive statistics

\begin{tabular}{llc}
\hline Variables & Mean & Standard deviation \\
\hline Women, $N(\%)$ & $102(87.9)$ & \\
Age & 48.11 & 12.03 \\
QL-Index & 6.96 & 2.99 \\
Mental turmoil & & \\
HAM-D & 14.14 & 11.03 \\
HAM-D > 15, $N(\%)$ & $33(28.4)$ & \\
BHS & 7.36 & 4.75 \\
BHS > 9, $N(\%)$ & $40(34.6)$ & \\
MINI suicide intent & $10(8.6)$ & \\
SSS part 1 & 0.13 & 0.25 \\
SSS part 2 & 0.18 & 0.28 \\
SSS total raw score & 0.15 & 0.24 \\
TEMPS-A & & \\
Hypertimic & 9.36 & 4.23 \\
Irritability & 5.28 & 3.36 \\
Dys-cyc-anx & 31.56 & 12.34 \\
IPQ & & \\
Symptoms & 7.10 & 2.72 \\
Timeline acute/chronic & 0.46 & 0.17 \\
Timeline cyclical/episodic & 0.54 & 0.23 \\
Consequences & 0.43 & 0.15 \\
Personal control & 0.32 & 0.12 \\
Treatment control & 0.48 & 0.12 \\
Illness coherence & 0.47 & 0.18 \\
Emotional representation & 0.39 & \\
\hline
\end{tabular}

timeline acute/chronic $(r=0.21 ; P<0.01)$, consequence $(r=.28 ; \quad P<0.01)$ and emotional representation $(r=0.43 ; P<0.01)$.

When evaluating the relationship between mental turmoil and illness perception, 18 of the 40 correlations were statistically significant (see Table 2). Thus, patients with higher mental turmoil have lower quality of life, with the strongest effect for depression and suicidal intent as measured via the SSS.

\section{Multivariate analysis}

To evaluate multivariate associations among variables, we performed a hierarchical multivariate regression analysis with quality of life as dependent variable (see Table 3). The analysis indicated that only the model with the mental turmoil variables fits data (Model 4: adjusted $R^{2}=0.36$; $R^{2}$ Change $=0.36 ; \mathrm{F}$ change $=5.98[d f: 5,35], P<0.01$; $F=2.67$ [df: 18,35], $P<0.01)$. Models with sociodemographic variables (Model 1: adjusted $R^{2}=-0.01 ; R^{2}$ change $=0.02 ; F$ change $=0.64[d f: 2,51], P=0.53$; $F=0.64[d f: 2,51], P=0.53)$, illness perception dimensions (Model 2: adjusted $R^{2}=-0.03 ; R^{2}$ change $=0.14$; 


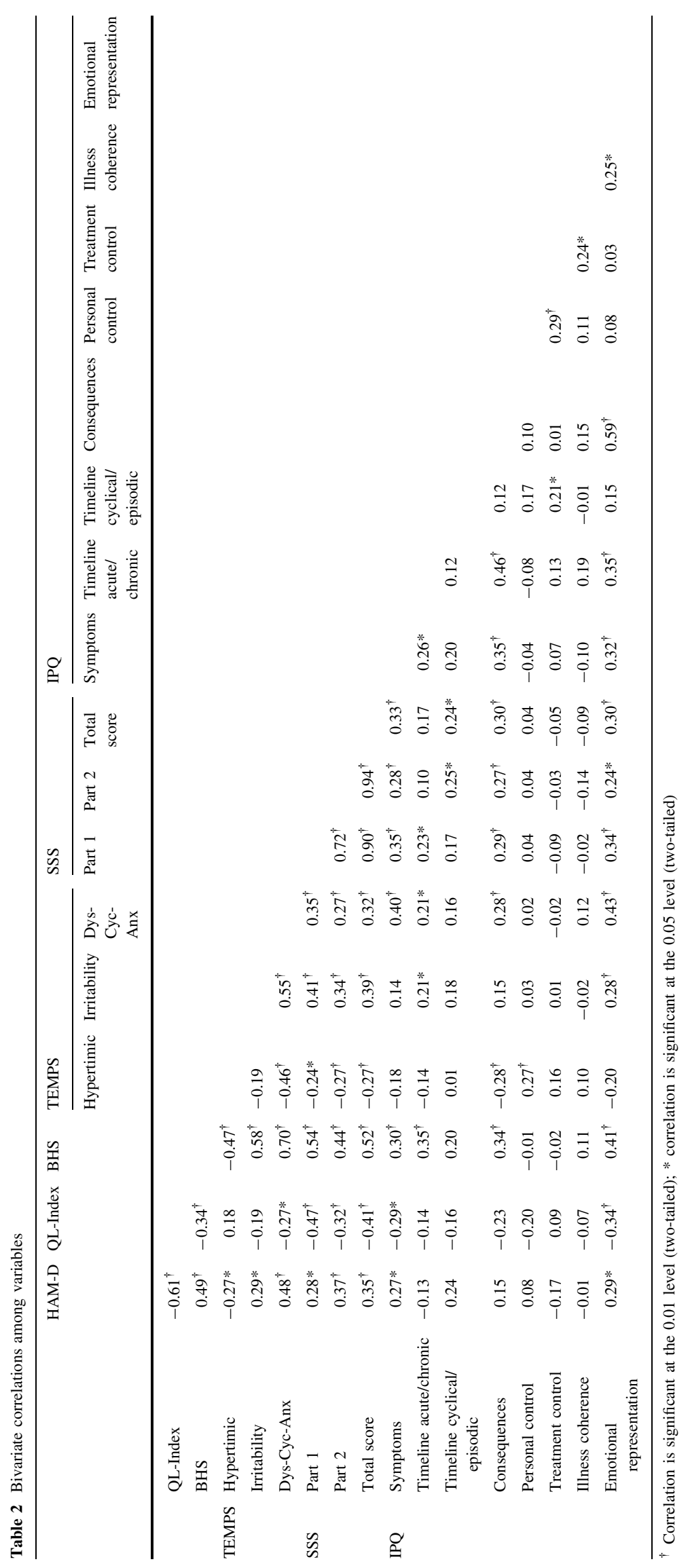


Table 3 Multivariate association among variables

\begin{tabular}{|c|c|c|c|c|c|c|c|c|}
\hline Step variables & $\begin{array}{l}1 \\
\text { Standardized } \\
\text { regression coefficient }\end{array}$ & $\begin{array}{l}2 \\
\text { Standardized } \\
\text { regression coefficient }\end{array}$ & \multicolumn{2}{|c|}{$\begin{array}{l}3 \\
\text { Standardized } \\
\text { regression coefficient }\end{array}$} & $\begin{array}{l}4 \\
\text { Standardized } \\
\text { regression coefficient }\end{array}$ & \multicolumn{3}{|c|}{$\begin{array}{l}\text { Final } \\
\text { Standardized } \\
\text { regression coefficient }\end{array}$} \\
\hline Age & $-0.15 \mathrm{NS}$ & $-0.10 \mathrm{NS}$ & $-0.14 \mathrm{NS}$ & & $-0.09 \mathrm{NS}$ & & & \\
\hline Sex & $0.03 \mathrm{NS}$ & $0.13 \mathrm{NS}$ & $0.14 \mathrm{NS}$ & & $0.16 \mathrm{NS}$ & & & \\
\hline Symptoms & & -0.14 & $-0.20 \mathrm{NS}$ & & $0.001 \mathrm{NS}$ & & & \\
\hline $\begin{array}{l}\text { Timeline acute/ } \\
\text { chronic }\end{array}$ & & $0.10 \mathrm{NS}$ & $0.13 \mathrm{NS}$ & & $0.02 \mathrm{NS}$ & & & \\
\hline $\begin{array}{l}\text { Timeline cyclical/ } \\
\text { episodic }\end{array}$ & & $-0.11 \mathrm{NS}$ & $-0.05 \mathrm{NS}$ & & $0.08 \mathrm{NS}$ & & & \\
\hline Consequences & & $-0.14 \mathrm{NS}$ & $-0.10 \mathrm{NS}$ & & $0.14 \mathrm{NS}$ & & & \\
\hline Personal control & & $-0.13 \mathrm{NS}$ & $-0.17 \mathrm{NS}$ & & $-0.18 \mathrm{NS}$ & & & \\
\hline Treatment control & & $0.11 \mathrm{NS}$ & $0.12 \mathrm{NS}$ & & $-0.02 \mathrm{NS}$ & & & \\
\hline Illness coherence & & $-0.08 \mathrm{NS}$ & $-0.14 \mathrm{NS}$ & & $-0.17 \mathrm{NS}$ & & & \\
\hline $\begin{array}{l}\text { Emotional } \\
\text { representation }\end{array}$ & & $-0.12 \mathrm{NS}$ & $-0.06 \mathrm{NS}$ & & $-0.11 \mathrm{NS}$ & & & \\
\hline Hypertimic & & & $0.28 \mathrm{NS}$ & & $0.31 \mathrm{NS}$ & & & \\
\hline Irritability & & & $-0.10 \mathrm{NS}$ & & $-0.25 \mathrm{NS}$ & & & \\
\hline Dys-Cyc-Anx & & & $0.09 \mathrm{NS}$ & & $0.16 \mathrm{NS}$ & & & \\
\hline HAM-D & & & & & $-0.26 \mathrm{NS}$ & & & \\
\hline BHS & & & & & $0.07 \mathrm{NS}$ & & & \\
\hline $\begin{array}{l}\text { MINI suicide } \\
\text { intent }\end{array}$ & & & & & $-0.55^{\dagger}$ & -0.5 & & \\
\hline SSS part 1 & & & & & $-0.17 \mathrm{NS}$ & & & \\
\hline SSS part 2 & & & & & $0.11 \mathrm{NS}$ & & & \\
\hline $\begin{array}{l}\text { SSS total raw } \\
\text { score }\end{array}$ & & & & & - & & & \\
\hline Models indeces step & Adjusted $R^{2}$ & $R^{2}$ change & $F$ change & $d f 1, d f 2$ & $P$ & $F$ & $d f 1 / d f 2$ & $P$ \\
\hline 1 & -0.01 & 0.02 & 0.64 & 2,51 & $<0.53$ & 0.64 & 2,51 & $<0.53$ \\
\hline 2 & -0.03 & 0.14 & 0.93 & 8,43 & $<0.50$ & 0.87 & 10,43 & $<0.57$ \\
\hline 3 & -0.04 & 0.05 & 0.85 & 3,40 & $<0.47$ & 0.86 & 13,40 & $<0.60$ \\
\hline 4 & 0.36 & 0.36 & 5.98 & 5,35 & $<0.01$ & 2.67 & 18,35 & $<0.01$ \\
\hline Final & 0.31 & 0.32 & 38.29 & 1,82 & $<0.01$ & 38.29 & 1,82 & $<0.01$ \\
\hline
\end{tabular}

NS not significant; ${ }^{\dagger}$ significant at $P<0.01$

$F$ change $=0.93 \quad[d f: 8,43], \quad P=0.50 ; \quad F=0.87 \quad[d f$ : 10,43], $P=0.57$ ), and temperament dimensions (Model 3: adjusted $R^{2}=-0.04 ; R^{2}$ change $=0.05 ; F$ change $=0.85$ [df: 3,40], $P=0.47 ; \quad F=0.86 \quad[d f: 13,40], P=0.60)$ failed to produce good fit. Only one variable was associated with quality of life: the MINI suicidal intent (standardized regression coefficient $=-0.55 ; \quad t=-3.06 ; \quad P<0.01)$. Finally, we computed a model with only this one significant variable. This model explained $31 \%$ of the variability of the QL-Index (Final model: adjusted $R^{2}=0.31 ; R^{2}$ change $=0.32 ; F=38.29 \quad[d f: 1,82], P<0.01)$. Thus, when controlling for the presence of other variables, only mental turmoil was significantly associated with the quality of life.

\section{Discussion}

The aim of the present study was to investigate the quality of life, temperament, suicide risk and depression in patients suffering from $\mathrm{CDH}$ with $\mathrm{MOH}$. We found that suicide risk played a central role in affecting the quality of life of the patients in this study. The study also reported the first evidence regarding temperament in patients suffering from chronic daily headache with $\mathrm{MOH}$.

Suicide is one of the world's largest public health problems, accounting for approximately 1 million lives lost annually. This represents a global mortality rate of 16 per 100,00 , or one death every 40 seconds. As one of the leading causes of death worldwide, particularly in younger 
people, it profoundly affects individuals, families, workplaces, neighbourhoods and societies. The economic costs associated with suicide and self-inflicted injuries are estimated to be in billions of dollars [42]. According to the World Health Organization, suicide is a huge but preventable public health problem [42].

Likewise, $\mathrm{CDH}$ is a source of great distress, with impairment of pleasure and working activities. Decreased quality of life can result in hopelessness and despair, and suicidal wishes can easily emerge. Of some importance is the close similarity between headache and mental turmoil. Both refer to pain, in the head and in the psyche, respectively. However, this symbolic association may have a basis in reality. Although Shneidman [43] admitted that each suicide is a multifaceted event, that biological, cultural, sociological, interpersonal, intrapsychic, logical, philosophical, conscious and unconscious elements are always present, he suggested that the essential nature of suicide is psychological, meaning that each suicidal drama occurs in the mind of a unique individual. It is widely acknowledged in suicidology that suicidal individuals are experiencing psychological pain or suffering and that suicide may be, at least in part, an attempt to escape from this suffering.

Shneidman [44, 45] coined the term "psychache" to describe this pain. Psychache is the hurt, anguish, or ache that takes hold of the mind, the pain of excessively felt shame, guilt, fear, anxiety, loneliness, angst and dread of growing old or of dying badly. Suicide is functional because it abolishes the pain for the individual. Suicide occurs when the psychache is deemed by that individual to be unbearable. It is an escape from intolerable suffering. One may wonder if this assumption is valid also for chronic daily headache and that suicide risk may increase when physical pain is higher than a given individual's threshold.

Although both $\mathrm{CDH}$ and depression seem to function as obstacles in the lives of those that are affected by them, they do not have any other outwardly symptoms in common. Many clinical studies, however, have noted the degree to which chronic daily headache seemed to be comorbid with other anxiety and affective disorders. It seems that this association of migraines with other types of disorders is important because, if the association is strong enough, the proper types of treatment available will have to be tested for their effects on the other comorbid condition. For example, $\mathrm{CDH}$ can be treated with many different types of drugs, and so for someone who experiences $\mathrm{CDH}$ and has also been diagnosed with Major Depressive Disorder (MDD), it would be important to avoid drug treatments, such as beta-blockers, which produce side effects such as drowsiness, fatigue, lethargy, sleep disorders, nightmares, depression, memory disturbance, and hallucinations [46]. This would run the risk of increasing the severity of the depressive symptoms. Instead, tricyclic antidepressants (TCAs) should be used, which would treat both the migraine and the depression.

There have been studies to see if this link between CDH and anxiety/affective disorders was substantial and to determine its cause. Breslau, Merikangas, and Bowden [47] thought that it would be important to first make sure that this link could actually be found in a population-based study. In fact, it seemed possible that the association between migraines and these various disorders in a clinical setting might be simply due to the possibility that patients suffering from multiple disorders might be more inclined to seek treatment. If this were true, then a population-based study would find much less of an association than would a clinical-based study. However, the results of the population-based study showed that the association was in fact genuine.

In these research papers, the term mood disorder was considered interchangeable with the term affective disorder. The main types of affective disorders that were examined were major depression, dysthymia, bipolar disorder and cyclothymia. Dysthymia is a type of chronic depressed or irritable mood disorder, and cyclothymia is a mild form of bipolar II disorder, characterized by major depressive and hypomanic episodes. It was found that dysthymia, MDD, manic episodes and bipolar spectrum disorders were more common in those who also experienced migraines than in those who did not. What was even more interesting, is that they discovered that there is a difference in the extent of comorbidity of migraine and affective disorders between those who experienced migraine with aura and those who experienced migraine without aura. Migraine patients with aura seemed to have a higher prevalence of each of the affective disorders. Specifically, "major depression was by far the most common affective disorder in persons with migraine with aura and migraine without aura" [47]. The next step was to try and explain this strong probability of coexistence. Two possible explanations were that (1) one condition might be causing the other, or (2) they might share genetic or environmental etiological factors. For example, it seems plausible that the extreme pain experienced during a migraine might cause one to feel completely powerless over one's own life, and thus feel depressed. To test if this was the cause of the association between migraine and depression, people were seen once and then again three and a half years later. The result showed that there is both a relative risk of developing MDD after experiencing migraine and of developing migraine after experiencing MDD. This makes the previously stated hypothesis about migraine causing depressive symptoms seem less plausible, since MDD also raises the risk of developing migraine. Thus there must be some other explanation for their comorbid association. 
Next, families were studied to see if the association between migraine and depression was transmitted, indicating genetic factors. However, it was found that there was little cross-transmission of the pure forms of the two disorders. If the association between the two disorders was causal, then relatives of a family member with migraine, for example, should have a higher probability of developing MDD only if he/she also had migraines. The test results in this specific study did confirm this type of association. There were no significant findings indicating a relative of someone with migraine developing MDD without also having migraine.

Some earlier experiments on the association between migraine and various psychiatric disorders investigated their relationship to suicidal thoughts and actions. Breslau and Davis [48] reported that people with migraine had a higher lifetime rate of suicide attempts than those with no history of migraine. In addition, those with MDD and migraine had a higher rate of suicide attempts than those with only Major Depressive Disorder (MDD): 31.8 and $16.5 \%$, respectively. This association of suicide attempts with migraine was found to be specific to migraine with aura. Breslau [49] found that the association of migraine with aura and suicide attempt was not necessarily due to a coexisting MDD. It may at first seem strange to discuss suicidal attempts outside of the realm of MDD, but it was stated in this paper that risk factors for suicide attempts, outside of MDD, can include heavy use of alcohol and other psychoactive substances, as well as a family history of suicidal behaviours. Thus, it was stated that migraine with aura alone was associated with significantly high rates of suicidal tendencies, and that the risks of suicidal inclinations associated with migraine with aura and MDD was much higher than that for those with only MDD. It was proposed that serotonin abnormalities might be the one factor that MDD, suicide and migraine all have in common, and that this would explain the associations of migraine with aura to suicide, because migraine with aura has been found to be accompanied by more pronounced serotonin imbalances than migraine without aura.

Abbate-Daga et al. [50] found that patients suffering from migraine show more depressive symptoms, difficult anger management with a tendency to hypercontrol, and a distinctive personality profile with high harm avoidance, high persistence and low self-directedness. When a logistic regression was performed, the only significant predictors of migraine were temperament variables. Their results suggest that the personality traits and the psychosomatic mechanisms of migraine patients may make them vulnerable to stress and less skilled in coping with pain. These traits correlate with dysregulated neurotransmitter systems which may also be part of the psychobiological components of personality, depressive disorders and migraine itself.
Sánchez-Román et al. [51] investigated the personality profile of a sample of Mexican patients with migraine using the Temperament and Character Inventory (TCI). They used two healthy control groups and a third group of nonmigraine pain controls. One hundred and forty two subjects with migraine, 108 healthy blood donors, 269 young healthy controls and 30 patients with non-migraine pain (NMP) were included in the study. They found that patients with chronic pain share some of the personality features of patients with migraine, but their TCI profile could be indicative of cluster $\mathrm{C}$ avoidant personality. Blood donors were shown to have more energy, with a tendency to help other people and be more optimistic. The results support serotoninergic involvement as explaining the physiopathology of migraine.

The strength of the present study is the systematic clinical interviews for clinical data and the use of widely used and validated psychometric instruments. There are limitations to the generalization of the present results. First, the relatively small sample sizes may affect the replicability of results. Second, although we investigated psychiatric variables, we did not make specific diagnoses. Of note, for instance, is the fact that the Hamilton Depression Rating Scale is not a tool for diagnosing depression but rather to determine a depressive dimension.

In conclusion, we stress the need to assess suicide risk carefully in patients suffering from $\mathrm{CDH}$ and $\mathrm{MOH}$. Obviously, more work is required in this area and future research should aim to compare groups with different forms of headache.

\section{Conflict of interest None.}

\section{References}

1. Headache Classification Subcommittee of the International Headache Society (2004) The international classification of headache disorders, 2nd edn. Cephalalgia 24:S9-S160

2. Karenberg A, Leitz C (2001) Headache in magical and medical papyri of ancient Egypt. Cephalalgia 21:911-916

3. De Filippis S, Salvatori E, Farinelli I, Coloprisco G, Martelletti P (2007) Chronic daily headache and medication overuse headache: clinical read-outs and rehabilitation procedures. Clin Ter 158:343-347

4. Atasoy HT, Atasoy N, Unal AE et al (2005) Psychiatric comorbidity in medication overuse headache patients with pre-existing headache type of episodic tension-type headache. Eur J Pain 9:285-291

5. Boes CJ, Capobianco DJ (2005) Chronic migraine and medication-overuse headache through the ages. Cephalalgia 25:378-390

6. Radat F, Creac'h C, Swendsen JD et al (2005) Psychiatric comorbidity in the evolution from migraine to medication overuse headache. Cephalalgia 25:519-522

7. de Filippis S, Salvatori E, Coloprisco G et al (2005) Headache and mood disorders. J Headache Pain 6:250-253

8. Cupini LM, Calabresi P (2005) Medication-overuse headache: pathophysiological insights. J Headache Pain 6:199-202 
9. Bag B, Hacihasanoglu R, Tufekci FG (2005) Examination of anxiety, hostility and psychiatric disorders in patients with migraine and tension-type headache. Int J Clin Pract 59:515-521

10. Perozzo P, Savi L, Castelli L et al (2005) Anger and emotional distress in patients with migraine and tension-type headache. $\mathbf{J}$ Headache Pain 6:392-399

11. Jacobson SA, Folstein MF (2003) Psychiatric perspectives on headache and facial pain. Otolaryngol Clin North Am 36:11871200

12. Wacogne C, Lacoste JP, Guillibert E et al (2003) Stress, anxiety, depression and migraine. Cephalalgia 23:451-455

13. Ong JC (2004) An exploration of the psychophysiology of negative stimuli among headache sufferers in a scheduled-waiting, pictureviewing task. Dissertation-Abstracts-International 65:1035

14. Leistad RB, Sand T, Westgaard RH et al (2006) Stress-induced pain and muscle activity in patients with migraine and tensiontype headache. Cephalalgia 26:64-73

15. Leistad RB, Nilsen KB, Stovner LJ et al (2008) Similarities in stress physiology among patients with chronic pain and headache disorders: evidence for a common pathophysiological mechanism? J Headache Pain 9:165-175

16. Akiskal HS, Brieger P, Mundt $C$ et al (2002) Temperament und affektive Störungen: Die TEMPS-A Skala als Konvergenz europäischer und US-amerikanischer Konzepte. Nervenarzt 73:262271

17. Akiskal HS (1996) The temperamental foundations of mood disorders. In: Mundt $\mathrm{CH}$ (ed) Interpersonal factors in the origin and course of affective disorders. Gaskell, London, pp 3-30

18. Kraepelin E (1921) Manic-depressive insanity and paranoia. E\&S Livingstone, Edinburgh

19. Kretschmer E (1936) Physique and character. Kegan, Paul, Trench Trubner and Co. Ltd, London

20. Akiskal HS, Khani MK, Scott-Strauss A (1979) Cyclothymic temperamental disorders. Psychiatr Clin North Am 2:527-554

21. Akiskal HS, Mallya G (1987) Criteria for the "soft" bipolar spectrum: treatment implications. Psychopharmacol Bull 23:6873

22. Akiskal HS, Akiskal KK (1992) Cyclothymic, hyperthymic and depressive temperaments as subaffective variants of mood disorders. In: Tasman A, Riba MB (eds) Annual review. American Psychiatric Press, Washington, DC, pp 43-62

23. Akiskal HS, Pinto O (2000) Soft bipolar spectrum: footnotes to Kraepelin on the interface of hypomania, temperament and depression. In: Marneros A, Angst J (eds) Bipolar disorders: 100 years after manic-depressive insanity. Kluwer, Dordrecht, pp 37-62

24. Akiskal HS, Akiskal KK (2005) TEMPS: temperament evaluation of Memphis, Pisa, Paris and San Diego. J Affect Disord $85: 1-2$

25. Schneider K (1958) Psychopathic personalities. Charles C. Thomas, Springfield

26. Akiskal HS (1998) Toward a definition of generalized anxiety disorder as an anxious temperament type. Acta Psychiatr Scand Suppl 393:66-73

27. Akiskal HS (1992) Delineating irritable-choleric and hyperthymic temperaments as variants of cyclothymia. J Pers Disord 6:326-342

28. Rihmer A, Rózsa S, Rihmer Z et al (2007) Affective temperament-types and suicidal behaviour. Eur Psychiatry 22:s244
29. Sheehan DV, Lecrubier Y, Sheehan KH et al (1998) The miniinternational neuropsychiatric Interview (M.I.N.I.): the development and validation of a structured diagnostic psychiatric interview for DSM-IV and ICD-10. J Clin Psychiatry 59 Suppl 20:22-33 quiz 34-57

30. Hamilton M (1967) Development of a rating scale for primary depressive illness. Br J Soc Clin Psychol 6:278-296

31. Hamilton M (1960) A Rating Scale for depression. J Neurol Neurosurg Psychiatry 23:56-62

32. Moss-Morris R, Weinman J, Petrie KJ et al (2002) The revised illness perception questionnaire (IPQ-R). Psychol Health 17:1-6

33. Spitzer WO, Dobson AJ, Hall J et al (1981) Measuring the quality of life of cancer patients: a concise QL-index for use by physicians. J Chronic Dis 34:585-597

34. Pompili M, Girardi P, Tatarelli R et al (2007) TEMPS-A (Rome): psychometric validation of affective temperaments in clinically well subjects in mid- and south Italy. J Affect Disord 107:63-75

35. Bedrosian RC, Beck AT (1979) Cognitive aspects of suicidal behavior. Suicide Life Threat Behav 9:87-96

36. Chandrasekaran R, Gnanaselane J (2005) Correlates of suicidal intent in attempted suicide. Hong Kong J Psychiatry 15:118-121

37. Wetzel RD, Margulies T, Davis R et al (1980) Hopelessness, depression, and suicide intent. J Clin Psychiatry 41:159-160

38. Beck AT, Steer RA, Kovacs M et al (1985) Hopelessness and eventual suicide: a 10-year prospective study of patients hospitalized with suicidal ideation. Am J Psychiatry 142:559-563

39. Beck AT, Brown G, Berchick RJ et al (1990) Relationship between hopelessness and ultimate suicide: a replication with psychiatric outpatients. Am J Psychiatry 147:190-195

40. Pompili M, Iliceto P, Lester D et al (2008) Beck Hopelessness Scale-Manuale. Organizzazioni Speciali, Firenze (in press)

41. Bagby RM, Ryder AG, Schuller DR et al (2004) The Hamilton depression rating scale: has the gold standard become a lead weight? Am J Psychiatry 161:2163-2177

42. World Health Organization (2004) Suicide huge but preventable public health problem, says WHO. World suicide prevention day-10 September. Cent Eur J Public Health 12:196, 200, 206

43. Shneidman ES (1993) Some controversies in suicidology: toward a mentalistic discipline. Suicide Life Threat Behav 23:292-298

44. Shneidman ES (1993) Suicide as psychache: a clinical approach to self-destructive behavior. Jason Aronson, Northvale

45. Shneidman ES (1993) Suicide as psychache. J Nerv Ment Dis $181: 145-147$

46. Silberstein SD, Lipton RB (1994) Overview of diagnosis and treatment of migraine. Neurology 44:S6-S16

47. Breslau N, Merikangas K, Bowden CL (1994) Comorbidity of migraine and major affective disorders. Neurology 44:S17-S22

48. Breslau N, Davis GC (1993) Migraine, physical health and psychiatric disorder: a prospective epidemiologic study in young adults. J Psychiatr Res 27:211-221

49. Breslau N (1992) Migraine, suicidal ideation, and suicide attempts. Neurology 42:392-395

50. Abbate-Daga G, Fassino S, Lo Giudice R et al (2007) Anger, depression and personality dimensions in patients with migraine without aura. Psychother Psychosom 76:122-128

51. Sanchez-Roman S, Tellez-Zenteno JF, Zermeno-Phols $F$ et al (2007) Personality in patients with migraine evaluated with the "Temperament and Character Inventory". J Headache Pain 8:94-104 